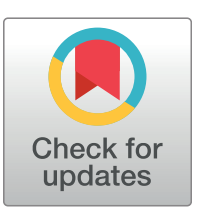

\section{OPENACCESS}

Citation: Trambaiolli LR, Tossato J, Cravo AM, Biazoli CE, Jr., Sato JR (2021) Subject-independent decoding of affective states using functional nearinfrared spectroscopy. PLOS ONE 16(1): e0244840. https://doi.org/10.1371/journal. pone. 0244840

Editor: Zhishun Wang, Columbia University, UNITED STATES

Received: April 3, 2020

Accepted: December 1, 2020

Published: January 7, 2021

Copyright: ๑ 2021 Trambaiolli et al. This is an open access article distributed under the terms of the Creative Commons Attribution License, which permits unrestricted use, distribution, and reproduction in any medium, provided the original author and source are credited.

Data Availability Statement: Data is available upon request because we are required to inform participants every time their data is shared. This is a standard procedure included in the consent form approved by the Ethics Committee of the Federal University of $A B C$. Future requests should be made to the local committee (cep@ufabc.edu.br).

Funding: This study was funded by the São Paulo Research Foundation (FAPESP): LRT received grant number 2015/17406-5, JT received grant number 2017/05225-1, JRS received grants
RESEARCH ARTICLE

\section{Subject-independent decoding of affective states using functional near-infrared spectroscopy}

\author{
Lucas R. Trambaiolli $\mathbb{D}^{1 *}$, Juliana Tossato ${ }^{2}$, André M. Cravo ${ }^{2}$, Claudinei E. Biazoli, Jr. $\mathbb{D}^{2}$, \\ João R. Sato
}

1 Division of Basic Neuroscience, McLean Hospital, Harvard Medical School, Boston, Massachusetts, United States of America, 2 Center for Mathematics, Computing and Cognition, Federal University of ABC, São Bernardo do Campo, São Paulo, Brazil

* lucasrtb@gmail.com

\section{Abstract}

Affective decoding is the inference of human emotional states using brain signal measurements. This approach is crucial to develop new therapeutic approaches for psychiatric rehabilitation, such as affective neurofeedback protocols. To reduce the training duration and optimize the clinical outputs, an ideal clinical neurofeedback could be trained using data from an independent group of volunteers before being used by new patients. Here, we investigated if this subject-independent design of affective decoding can be achieved using functional near-infrared spectroscopy (fNIRS) signals from frontal and occipital areas. For this purpose, a linear discriminant analysis classifier was first trained in a dataset (49 participants, $24.65 \pm 3.23$ years) and then tested in a completely independent one (20 participants, $24.00 \pm 3.92$ years). Significant balanced accuracies between classes were found for positive vs. negative $(64.50 \pm 12.03 \%, p<0.01)$ and negative vs. neutral $(68.25 \pm 12.97 \%, p<0.01)$ affective states discrimination during a reactive block consisting in viewing affective-loaded images. For an active block, in which volunteers were instructed to recollect personal affective experiences, significant accuracy was found for positive vs. neutral affect classification $(71.25 \pm 18.02 \%, p<0.01)$. In this last case, only three fNIRS channels were enough to discriminate between neutral and positive affective states. Although more research is needed, for example focusing on better combinations of features and classifiers, our results highlight fNIRS as a possible technique for subject-independent affective decoding, reaching significant classification accuracies of emotional states using only a few but biologically relevant features.

\section{Introduction}

Multivariate brain decoding (MBD) might allow the inference of mental states based solely on specific brain signals' features [1]. In comparison to conventional analytic methods, this approach has the advantage of considering the various brain regions simultaneously, thus 
number 2018/21934-5 and 2018/04654-9. The funders had no role in study design, data collection and analysis, decision to publish, or preparation of the manuscript.

Competing interests: The authors have declared that no competing interests exist. providing information on the neural networks level [2]. One particular application of MBD is to identify emotional or affective experiences, a critical step to develop brain-computer interfaces or neurofeedback protocols [3]. The clinical application of affective neurofeedback is of interest since it can putatively enable volitional control over abnormal neural activities or connectivity associated with emotional symptoms, potentially relieving symptom's severity $[4,5]$.

Conventional clinical neurofeedback approaches are based on subject-specific designs [4]. In other words, the patient is first submitted to an initial screening to create a calibration database for the neurofeedback algorithm and, later, starts the proper neurofeedback training. However, although reliable to the participant's neural data, this initial step leads to long sessions that might increase physical and mental exhaustion and, consequently, reduce the therapeutic benefits [6]. Thus, ideally, therapeutic affective neurofeedback should be trained using a set of volunteers and then applied with little or no calibration to a new individual. However, subject-independent identification of affective mental states remains an open technical challenge to neurofeedback implementation [1].

Subject-independent emotion classification has been previously reported on facial and body expressions [7, 8], and voice tone [7]. A critical limitation of those previous studies is that volunteers can easily handle the records used in order to achieve the desired result, even without changing their affective states. Moreover, such exclusively behavioral measures do not provide neural information, which is crucial to a properly designed neurofeedback system. Thus, investigations using neurophysiological records such as electrophysiology (EEG) and functional magnetic resonance imaging (fMRI) should be used in order to develop affective interfaces. Using EEG data, for example, different studies using a subject-independent design combined several features and classifiers to predict the affect experienced by new participants, achieving accuracies between 70 and 95\% [9-12]. Using fMRI data, accuracy varied from $60 \%$ to $80 \%$ depending on the number of voxels (from 2000 to 4000 ) included as predictors [13].

Another challenge for fostering clinical affective neurofeedback applications is the highcost and limited mobility of the so far most accurate imaging measurements, particularly fMRI [6]. In this context, functional Near-Infrared Spectroscopy (fNIRS) emerges as an attractive neuroimaging technique for affective decoding. This method is based on lowenergy light detectors and transmitters for measuring the light absorption through the cortical surface [14]. It makes it possible to investigate local changes in the concentrations of oxy and deoxyhemoglobin in response to functional brain activity, similarly to the widely applied fMRI BOLD effect [15]. Furthermore, fNIRS presents a good trade-off between spatial and temporal resolutions, with low susceptibility to instrumental and biological noise, and considerably lower cost and higher portability when compared to other non-invasive neuroimaging approaches [16]. Affective neuroscience experiments are benefited from fNIRS usage due to its reliability to record the prefrontal cortex activation, as well as allowing both strictly controlled and naturalistic emotion-related experimental designs [16-18]. To date, several studies showed a significant subject-specific offline prediction of affective states using different fNIRS protocols [19-23].

Using a fronto-occipital fNIRS setup, our research group achieved 80 to $95 \%$ of within-subject affective classification accuracy [22], and developed a functional subject-specific neurofeedback protocol [24]. Here, considering the above-mentioned technical challenges for subject-independent affective decoding, we aimed to evaluate whether these fronto-occipital fNIRS signals provide enough information to the subject-independent offline classification of affective states as a pilot investigation of the feasibility of fNIRS-based subject-independent affective decoding. To achieve this goal, we collected two completely independent datasets with reactive and active affective tasks. The reactive task was based on the visualization of a set of images from the International Affective Pictures System (IAPS) that were thought to induce 
positive, negative or neutral affective states [25]. Critically, those images were selected to balance the arousal dimension for the different valences. In the active task, participants were instructed to imagine personal situations with positive, negative or neutral affective contexts. We expected decoding performances higher than the chance level when comparing two different affective states in each task. Also, based on the affective-workspace model [26, 27], we expected the relevant information used in classification to come from nodes of neural networks mainly comprising frontal and occipital areas [28, 29].

\section{Methods}

Ethical approval for this study was obtained from the Federal University of ABC Ethics Committee.

\section{Participants}

Forty-nine healthy participants ( 25 female, $24.65 \pm 3.23$ years) were enrolled in the first part of the experiment. Inclusion criteria were no previous (self-reported) diagnosis of neurological (ICD-10: G00-G99) and/or psychiatric disorders (ICD-10: F00-F99), and normal or correctedto-normal vision. All participants were attending college or graduated and provided written informed consent to participate in this study.

Two years later, a second (and independent) sample of twenty healthy participants (10 female, $24.00 \pm 3.92$ years) was collected by a different researcher, and in a separate laboratory (at the same university). The inclusion criteria and the experimental protocol were equal to the first sample. However, considering that subjects, environmental noises, and experimenter bias are different between datasets, we treat them as two independent datasets. For both cases, ethical approval was obtained from the local Ethics Committee, and no payment was provided to the participants, according to the national rules.

\section{Functional NIRS acquisition}

fNIRS measurements were conducted using the NIRScout System (NIRx Medical Technologies, LLC. Los Angeles, California) using an array of optodes (12 light sources and 12 detectors) covering the prefrontal, temporal and occipital areas. Optodes were arranged in an elastic band, with nine sources and nine detectors positioned over the frontal and temporal regions, and three sources and three detectors over the occipital region. Four positions of the International 10-20 System were adopted as reference points during the setup: sensors 1 and 9 were positioned approximately over the $\mathrm{T} 7$ and $\mathrm{T} 8$ locations, respectively, while the $\mathrm{Fpz}$ and $\mathrm{Oz}$ positions were in the center of channels 5-5 and 11-11, respectively, as shown in Fig 1 . The source-receptor distance was $30 \mathrm{~mm}$ for adjacent optodes, and the used wavelengths were 760 and $850 \mathrm{~nm}$. Signals obtained from the thirty-two channels were measured with a sampling frequency of $5.2083 \mathrm{~Hz}$ (determined by the maximum sampling frequency of the equipment$62.5 \mathrm{~Hz}$-divided by the number of sources-12) using the NIRStar 14.0 software (NIRx Medical Technologies, LLC. Los Angeles, California).

\section{Experimental protocol}

During the test, participants sat in a padded chair with armrest, positioned 1-meter distance in front of a monitor. They were asked to remain relaxed, with hands within sight resting on the armrests or the table. They were also requested to avoid eye movements, as well as any body movement. The recording room remained dark during registration and the subject used earplugs. 
Schematic representation of optode locations:
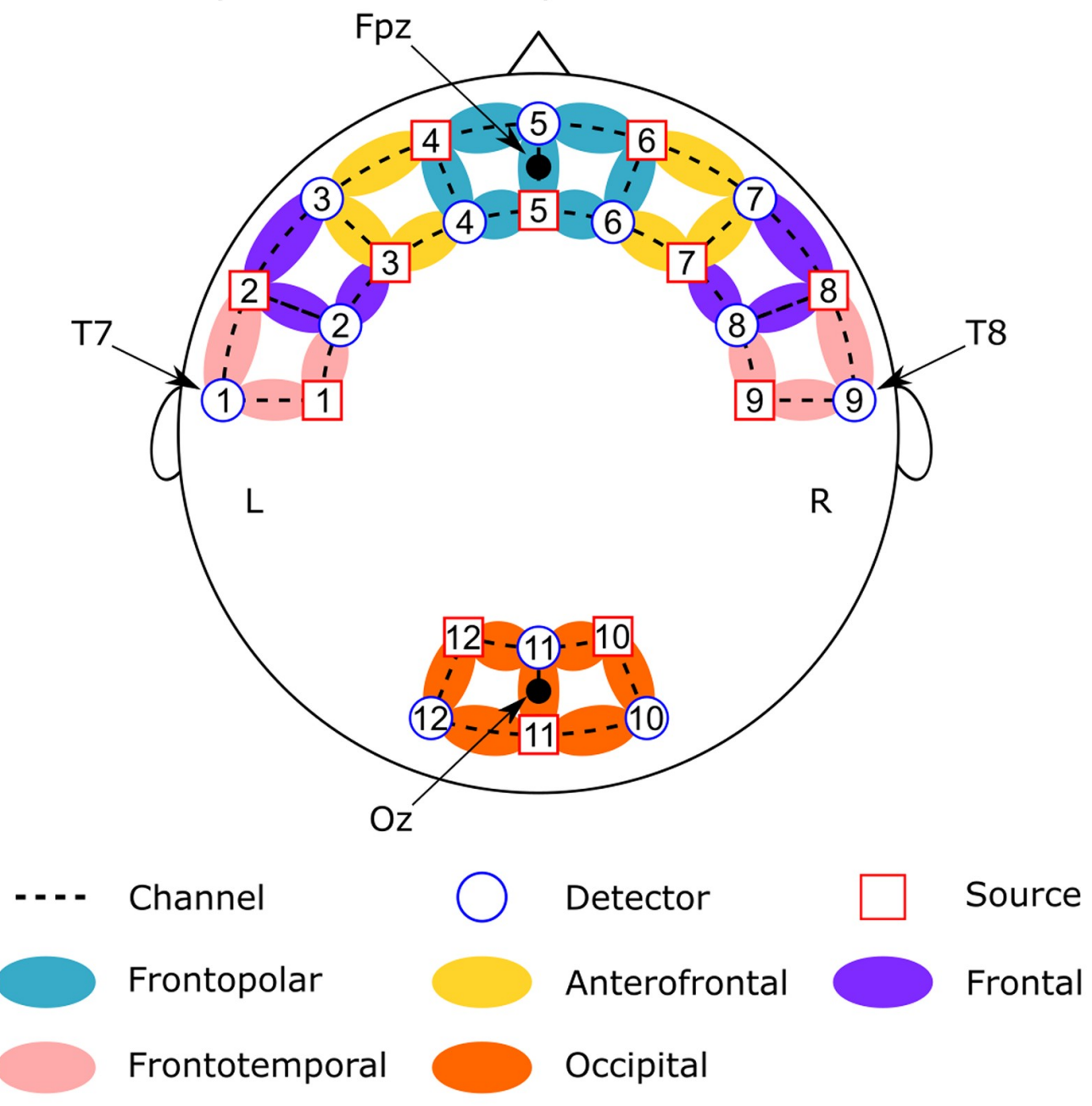

\section{Real optode configuration:}
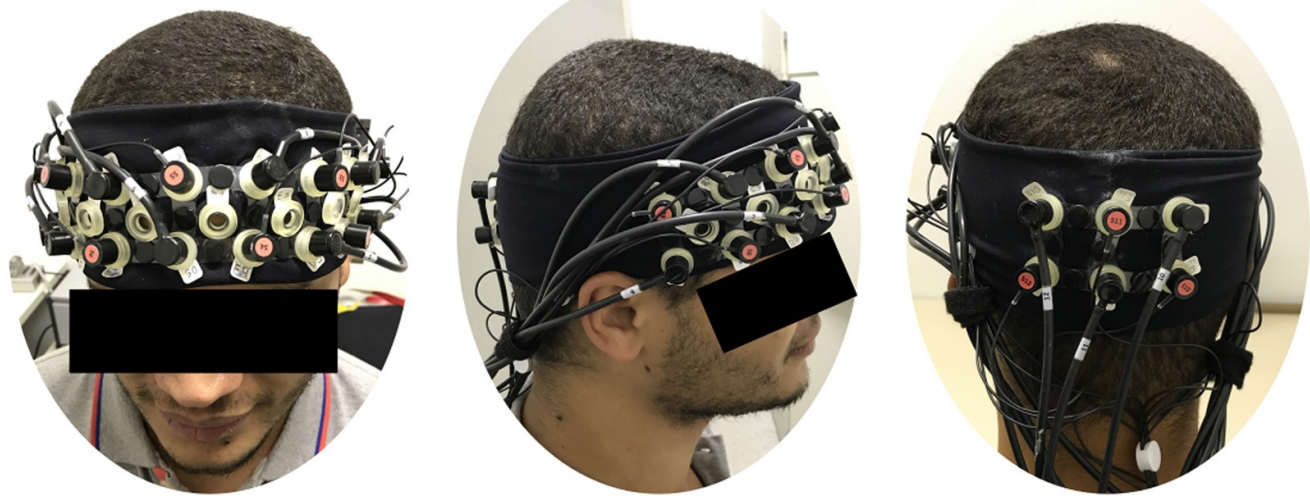

Fig 1. Channel configuration. Red circles represent sources; blue circles represent the detectors and dotted lines the channels. Colors show channel positions according to the International 10-20 system.

https://doi.org/10.1371/journal.pone.0244840.g001 
Each subject completed an eleven-point Likert mood scale immediately before and after the session, to evaluate the possible influence of mood on our results. This test quantifies her/his agitation, strength, confusion, agility, apathy, satisfaction, worry, perspicacity, stress, attention, capacity, happiness, hostility, interest, and introspection [30].

Reactive task. For the reactive task, we used images available on the international affective picture system (IAPS) catalog [25]. First, images were filtered according to their average values of arousal and then were ranked according to their valence values. We selected the 30 pictures with higher average values of valence, the 30 with lowest values and 60 of intermediate values, as follows:

- Positive pictures (Valence $=7.884 \pm 0.220$; Arousal $=5.036 \pm 0.448): 1811,2057,2080,2209$, $5210,5830,7200,2040,2058,2091,2340,5700,5833,7330,1440,2045,2070,2150,2347$, $5825,5910,7502,1710,2050,2071,2165,2550,5829,5982,8420$;

- Negative pictures ( $\mathrm{V}=2.007 \pm 0.183 ; \mathrm{A}=5.549 \pm 0.339): 2375.1,3101,3261,9181,9322,9560$, $2703,3180,3301,9185,9326,9571,2095,2800,3191,3350,9253,9332,2205,3016,3225$, 9040, 9300, 9421, 2345.1, 3062, 3230, 9140, 9301, 9433;

- Neutral pictures ( $\mathrm{V}=5.234 \pm 0.060 ; \mathrm{A}=3.770 \pm 0.813)$ : 2122, 2514, 5520, 7019, 7182, 7550, 2191, 2635, 5531, 7021, 7207, 7632, 2211, 2702, 5532, 7043, 7242, 7830, 1122, 2308, 2745.1, $5533,7052,7248,8065,1350,2377,2850,5740,7053,7249,1616,2381,2870,5920,7058$, $7365,1675,2385,2880,6910,7062,7497,1820,2487,5395,7001,7080,7500,1908,2495$, $5471,7014,7090,7506,2102,2499,5510,7017,7100$.

This task consisted of twenty trials ( 5 for positive stimuli, 10 for neutral and 5 for negative). For the first 2 seconds of each trial, a white cross was presented in the center of a blank screen. During the next 30 seconds, a new figure was displayed every 5 seconds, totaling six randomly selected figures per trial corresponding to the desired affective class (Fig 2). Presenting a group of images with the same valence ensures the maintenance of cognitive engagement [31] and the required duration to achieve the peak of oxygen concentration change relative to baseline [32]. At the end of the trial, a new screen was presented asking the participant to assign a score from 1 to 9 for the subjective valence ( 1 -extremely negative valence; 9 -highly positive valence) and subjective arousal (1 -lower arousal; 9 -higher arousal) experiences. After this, a blank screen appears for a random duration between 2-4 seconds and participants were instructed to blink and/or move in this period but not in the other phases.

Active task. The active task (affective imagination) consisted of twenty trials ( 5 trials for positive affect, 5 for negative affect and 10 for resting with eyes open, also referred as neutral affect). Each trial started with a baseline period of a blank screen with a white cross in the center. After 2 seconds, the instruction (representing the desired emotion) appeared to the left of the display, remaining on the screen for $2 \mathrm{~s}$ (Fig 2). The instruction consisted of either a green arrow pointing up (positive affect), a red arrow pointing downward (negative affect) or a blue circle (neutral affect). For 30 seconds after the instruction was presented, the screen remained unchanged, corresponding to the participant's affective imagination period. At the end of the trial, a new screen was presented asking the participant to assign a score from 1 to 9 for the subjective valence (1 extremely negative valence; 9 -highly positive valence) and subjective arousal (1 -lower arousal; 9 -higher arousal) experiences. After this, a blank screen appears for a random duration between 2 to 4 seconds and participants were instructed to blink and/or move in this period.

\section{Data analysis}

Preprocessing. Preprocessing was performed using Matlab (Mathworks, MA, USA) with the nirsLAB v2014.12 toolbox (NIRx Medical Technologies, LLC. Los Angeles, California). 


\title{
Experimental sequence
}

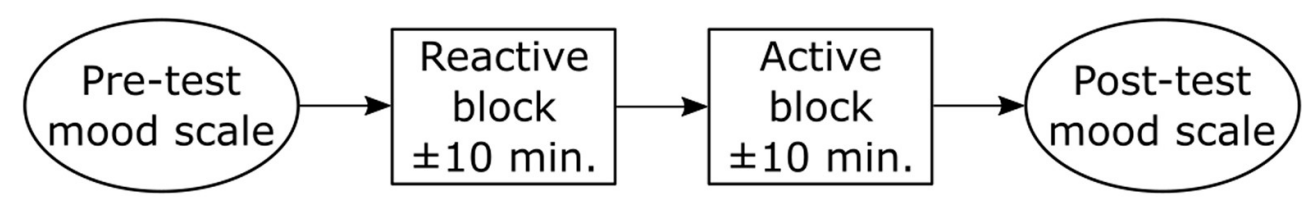

\author{
Block \\ structure
}
Reactive trial

\section{Positive trial Neutral trial Negative trial}

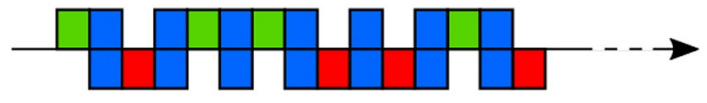

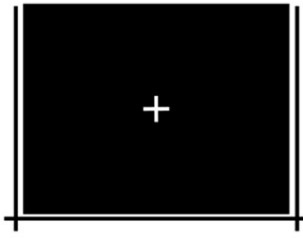

$0-2 s$

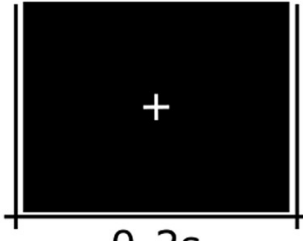

$0-2 s$
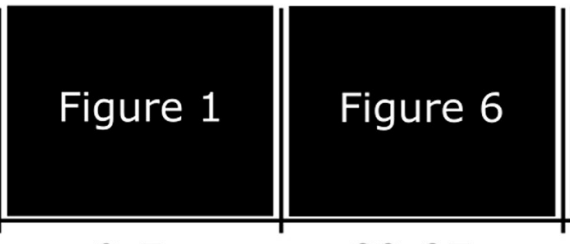

$2-7 s$
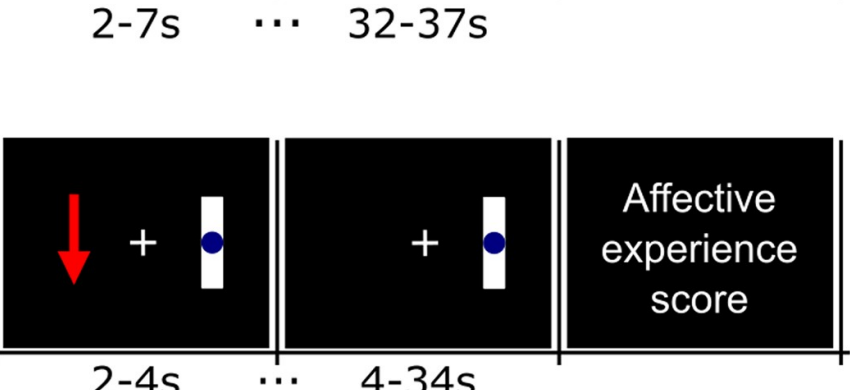

Fig 2. Visual stimuli order in reactive and active trials. The order of trials into the block is random, but always alternating neutral trials with positive and negative affect trials.

https://doi.org/10.1371/journal.pone.0244840.g002

Considering that Mayer waves-related noise around $0.1 \mathrm{~Hz}$ might interfere in the classification of fNIRS data [33, 34], we used a conservative frequency range in our analysis $(0.01-0.1 \mathrm{~Hz})(\mathrm{a}$ supplementary analysis with a broader filter is included in the S1 File). Thus, each participant's raw data were digitally band-pass filtered by a linear-phase FIR filter $(0.01-0.1 \mathrm{~Hz})$. Besides Mayer waves, this range also filters noises due to heartbeat $(0.8 \sim 1.2 \mathrm{~Hz})$ and respiration $(0.3$ $\mathrm{Hz})[35,36]$. Then, each wavelength was detrended by their respective whole length record (without segmentation), and variations in concentration of oxyhemoglobin and deoxyhemoglobin were calculated by the modified Beer-Lambert law (differential pathlength factor set to 7.25 and 6.38, respectively) [37]. Each concentration curve was then segmented into the $30 \mathrm{~s}$ of interest of each trial, for all studied conditions (Fig 3a).

The mean concentration of oxyhemoglobin and deoxyhemoglobin for each segment was calculated for each channel using the average of moving 2 s-window means with $50 \%$ overlap. Thus, each subject's database was composed of 64 features of average concentration (32 channels $\times 2$ chromophores) in 20 experimental conditions ( 10 neutral trials +5 positive trials +5 negative trials), for both tasks.

Machine learning procedure. To decode affective states across databases, we used the Linear Discriminant Analysis (LDA) implementation provided by the BCILAB toolbox [38]. To avoid overfitting the model to our dataset, the LDA was computed with its default parameters. This analysis was divided in two main steps represented in Fig 3b. 
a

Whole signal analysis

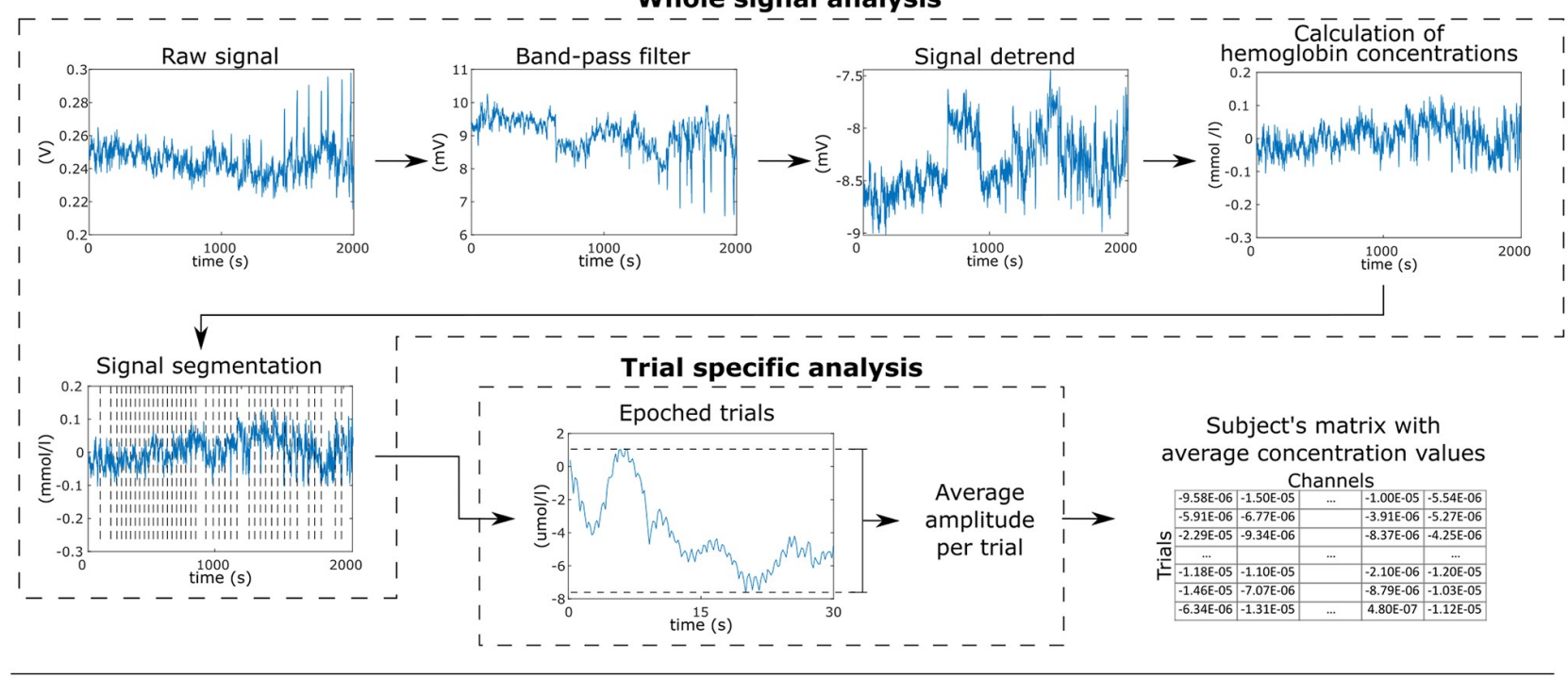

b

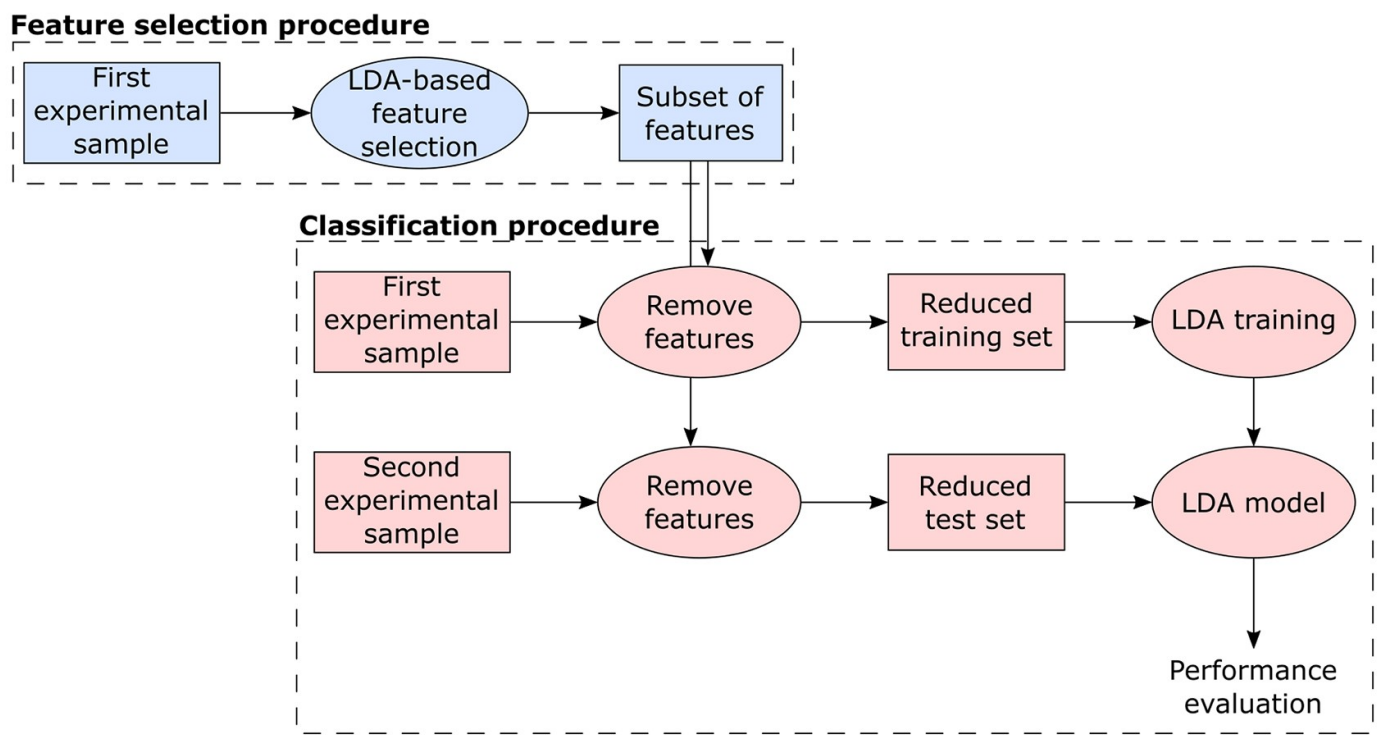

Fig 3. (a) Feature extraction procedure, showing steps from the whole raw signal until the creation of a feature table per subject. (b) Machine learning procedure: Initially, we performed an LDA-based feature selection using the first experimental sample ( 49 subjects). Then, based on these selected features, we created training and test sets for the LDA classifier using the first ( 49 subjects) and second ( 20 subjects) experimental samples, respectively.

https://doi.org/10.1371/journal.pone.0244840.g003

First, we performed an LDA-based feature selection using the whole feature space from the first experimental sample (49 participants) (steps in blue in Fig 3b). The LDA model searches for a linear projection of multivariate observations to univariate ones [39]. During the LDAbased feature selection, the eigenvalues of the within-class covariance matrix in the LDA can be used as a measure of feature relevance [40-44]. Thus, after sorting these eigenvalues, larger values provide the most discriminative information, while smaller values indicate less relevant features. This approach was successfully employed as a feature selection step in different neuroimaging studies [41, 43-45], even outperforming other classifier-based feature selection approaches [45]. In this study, we first ranked the absolute eigenvalues from all features, and then created different subsets selecting the best 5 to $100 \%$ (with steps of $5 \%$ ) features with 
highest eigenvalues (in other words, one subset using the top 5\% features, another subset using the top $10 \%$, and so on).

For each obtained feature subset, projections of the training and testing datasets described by the selected features only were built using the first ( 49 participants) and second ( 20 participants) experimental samples, respectively (steps in red in Fig $3 b$ ). Then, we evaluated the predictive performance on each corresponding test set. This approach was applied to evaluate affective conditions in pairs, following "Positive versus Neutral", "Negative vs. Neutral" and "Positive vs. Negative" combinations, for reactive and active tasks separately. Here we followed this binary approach since many current affective neurofeedback protocols are mainly based on two-states designs [24, 46-51].

Since the train and test sets are composed of two independent datasets, cross-validation analyses do not apply to this case. Thus, to evaluate the prediction performance in each comparison, we applied the trained model to decode data from each subject from the test set, and calculated accuracy as: (trials correctly classified from first class + trials correctly classified from second class)/2. This ratio avoids the potential unbalance of sample size in each comparison, and this random result should be $50 \%$.

Two complementary analysis are described in the S1 File: one repeating exactly the same procedure using a Support Vector Machine classifier; other using the LDA classifier in a leaveone-subject-out cross-validation including all 69 subjects.

Statistical analysis. Considering that the results here presented are sum-based proportions, and no ceiling-effect was observed, these data assume approximately normal distributions. Thus, here we applied parametric t-test to evaluate our results.

First, the subject scores of valence and arousal of the second database were evaluated by a two-sample $t$-test. The comparisons used the mean values of valence and arousal assigned during positive, negative and neutral trials. This procedure was repeated for both tasks. The $\mathrm{p}-$ values were Bonferroni corrected for multiple comparisons ( 2 subjective measures $\times 3$ comparisons of conditions $\times 2$ tasks).

To evaluate possible influences of mood fluctuations on the classification performance, we calculated the Spearman's correlation between the best classification accuracies in each block and the pre-experiment mood scores, as well as between the best classification accuracies and the delta (post-experiment minus pre-experiment) mood scores. P-values were Bonferroni corrected for 64 multiple comparisons (2 experiment blocks x 2 time points x 16 mood measures).

The significance of classification accuracy of each comparison was evaluated by a one sample t-test, comparing the prediction performance from all participants in this comparison against the chance level (50\%). Each result was independently adjusted by using the Bonferroni correction for 120 multiple comparisons (20 different percentages of features $\times 3$ comparisons of conditions $\times 2$ tasks).

\section{Results}

\section{Subjective scores of arousal and valence}

Table 1 presents the mean subjective scores of arousal and valence relative to the positive, negative and neutral conditions, during both active and reactive tasks. Moreover, the corrected pvalues of each comparison are also presented.

Valence scores presented significant differences for all the comparisons. It is relevant, however, that no significant difference of the arousal scores was found during "Positive vs. Negative" comparisons, as well as during "Positive vs. Neutral" comparison during the reactive task. 
Table 1. In the first half of the table, mean \pm standard-deviation of subjective scores (ranging from 1 to 9 ) assigned by each subject and for each trial of the second database during negative, neutral and positive trials. In the second half, p-values of the comparison between the scores assigned for each trial type.

\begin{tabular}{|c|c|c|}
\hline \multicolumn{3}{|c|}{ Mean subjective scores } \\
\hline & Valence & Arousal \\
\hline \multicolumn{3}{|c|}{ Reactive task } \\
\hline Negative & $2.52 \pm 1.38$ & $6.05 \pm 1.34$ \\
\hline Neutral & $5.11 \pm 0.73$ & $2.71 \pm 1.29$ \\
\hline Positive & $6.79 \pm 0.92$ & $4.82 \pm 1.60$ \\
\hline \multicolumn{3}{|c|}{ Active task } \\
\hline Negative & $2.57 \pm 0.97$ & $5.85 \pm 1.71$ \\
\hline Neutral & $4.84 \pm 0.24$ & $2.09 \pm 1.39$ \\
\hline Positive & $7.20 \pm 0.77$ & $5.67 \pm 1.75$ \\
\hline \multicolumn{3}{|c|}{ Statistical comparisons (p-values) } \\
\hline & Valence & Arousal \\
\hline \multicolumn{3}{|c|}{ Reactive task } \\
\hline Positive vs. Negative & $<0.01$ & 0.14 \\
\hline Positive vs. Neutral & 0.02 & 1.00 \\
\hline Negative vs. Neutral & $<0.01$ & $<0.01$ \\
\hline \multicolumn{3}{|c|}{ Active task } \\
\hline Positive vs. Negative & $<0.01$ & 1.00 \\
\hline Positive vs. Neutral & $<0.01$ & $<0.01$ \\
\hline Negative vs. Neutral & $<0.01$ & $<0.01$ \\
\hline
\end{tabular}

https://doi.org/10.1371/journal.pone.0244840.t001

No significant correlations were found between pre-experiment mood scores and classification accuracies, or delta mood scores and classification accuracies. These results suggest that the classification results resulted from temporary affective changes induced during the respective trial, and not due to mood fluctuations not related to the experiment.

\section{Classification results}

Boxplots describing the LDA accuracy are presented in Fig 4. Classification accuracy for the reactive task significantly exceeded chance level in "Positive vs. Negative" comparisons, with highest mean result as $64.50 \pm 12.03 \%$ (mean \pm standard-deviation, $\mathrm{p}<0.01$ ) using $20 \%$ of features (12 channels), and in "Negative vs. Neutral" comparisons (68.25 $\pm 12.97 \%, \mathrm{p}<0.01,30 \%$ of features- 19 channels). No significant differences were found for "Positive vs. Neutral" comparisons.

For the active task, accuracies were greater than chance level in "Positive vs. Neutral" comparisons, and the highest mean accuracy was $71.25 \pm 18.02 \%$ ( $\mathrm{p}<0.01$, with maximum value for one single participant equals to $100 \%$ ) using only $5 \%$ of the features ( 3 channels). No significant differences were found for "Positive vs. Negative" and "Negative vs. Neutral" comparisons.

\section{Relevant channels}

In Fig 5, the channels selected in each of the highest significant results reported in Section 3.2 are shown alongside their respective weights assigned by the LDA classifier. The location of the channels follows the same order shown in Fig 1.

Among the "Positive vs. Negative" classification during the reactive task, the highest accuracy was achieved using 12 features. Nine of these features correspond to information about 

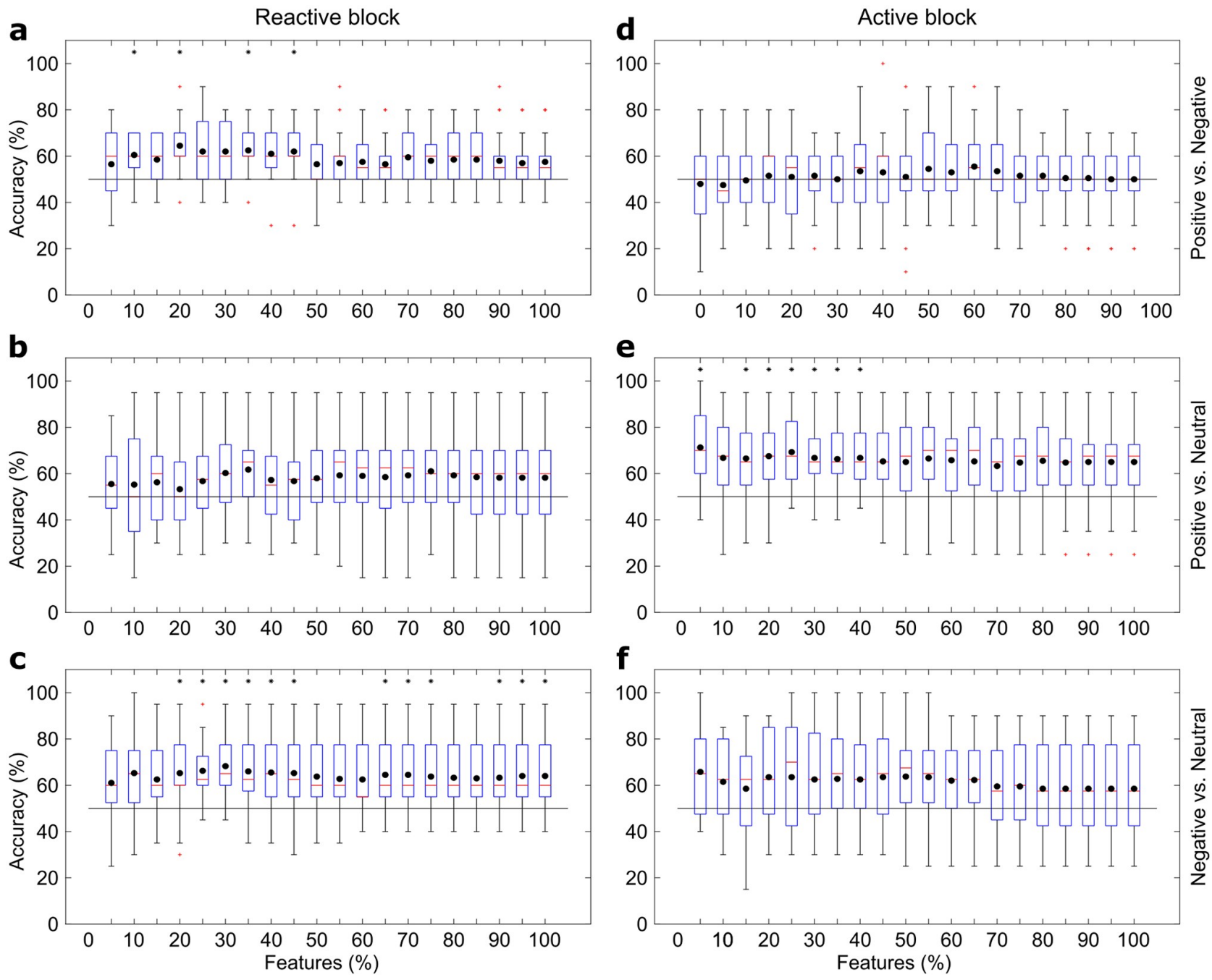

Fig 4. Box plots showing the results using the LDA classifier and different feature subsets. (a) presents the "Positive vs. Negative", (b) the "Positive vs. Neutral", and (c) the "Negative vs. Neutral" comparisons for reactive elicitation block, while (d-f) follows the same order for active elicitation block. Black dots present the means, red lines the medians, red crosses the outliers and black asterisks the statistical difference for chance level $(\mathrm{p}<0.05)$.

https://doi.org/10.1371/journal.pone.0244840.g004

the deoxyhemoglobin concentration. According to the 10-5 EEG electrode positioning system [52], four channels are approximately at the frontopolar area, two at the anterofrontal area, and three at the occipital area. Complementary, three features included information about the oxyhemoglobin concentration at the frontopolar (two channels) and the occipital (one) areas.

For the "Negative vs. Neutral" classification during the reactive task, 19 channels carried relevant information: six frontopolar, five anterofrontal, one frontal, and three occipital channels for the deoxyhemoglobin concentration; and two frontopolar and two occipital channels for the oxyhemoglobin concentration.

Finally, the "Positive vs. Neutral" decoding during the active task used only three channels to achieve the highest performance: two frontopolar and one anterofrontal. These channels provided information about the deoxyhemoglobin concentration. The corresponding averaged time series are shown in Fig 6, as well as the averaged values used as inputs to the LDA classifier. It is notable that the time series from second sample have higher variability, since the number of subjects (20) is smaller than the first sample (49). However, it is also notable that 

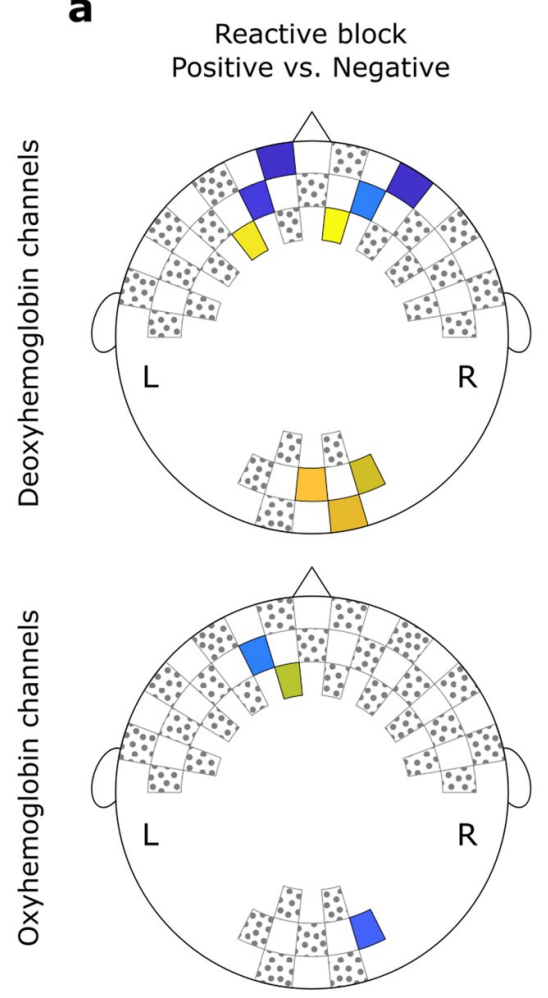

b
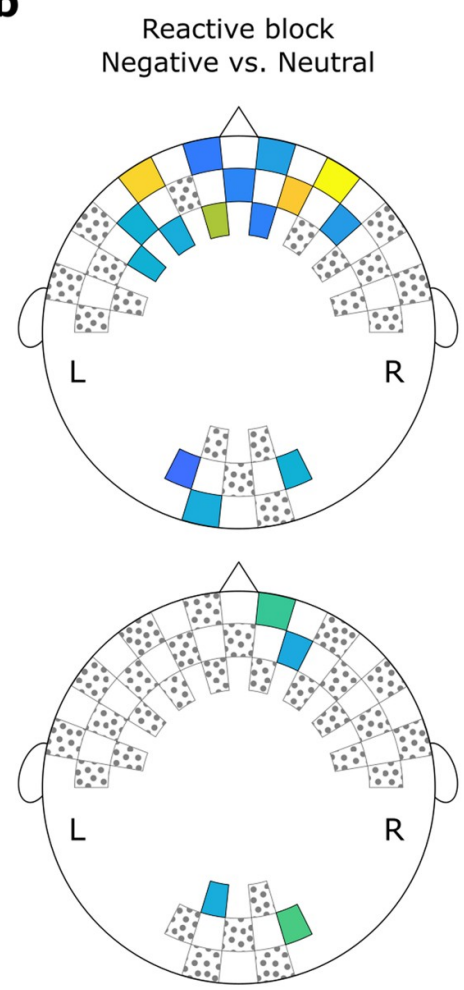

C
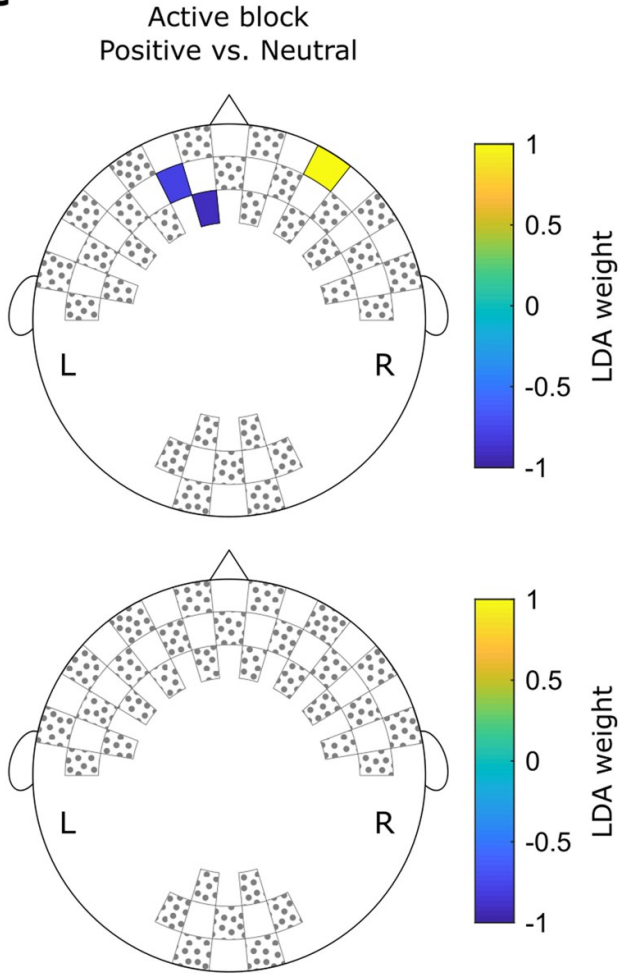

Fig 5. Weights assigned by the LDA classifier for each feature during the (a) "Positive vs. Negative" comparisons using $20 \%$ of features and (b) "Negative vs. Neutral" comparisons using $40 \%$ of features. In (c), the weights for each feature during the "Positive vs. Neutral" comparisons using $5 \%$ of features. Hottest colors indicate positive weights while cooler colors indicate negative weights. Channels filled with gray dots were not used during the test.

https://doi.org/10.1371/journal.pone.0244840.g005

the averaged features follow the same patterns in both samples, with positive trials presenting higher concentration levels in channel 6-7, and neutral trials presenting higher concentrations in channels 5-4 and 4-4. This is also consistent with the directions of the LDA weights showed in Fig $5 c$.

\section{Discussion}

\section{Affective decoding}

First, it is essential to highlight the absence of statistical significance in the subjective arousal score during the "Positive vs. Negative" comparisons. In addition to the significant difference in the valence scores, it suggests that any classification result in these comparisons is exclusively related to valence differences between positive and negative affect. Further, during both "Positive vs. Neutral" and "Negative vs. Neutral" comparisons, statistical differences were found for valence and arousal scores. It is expected that Positive and Negative present differences in arousal and valence when compared to absent affective content (Neutral). Some studies suggest valence and arousal not as two orthogonal dimensions, but as a single $\mathrm{V}$-shaped dimension [53]. Accordingly, these two dimensions cannot be separated in most of the emotional stimuli systems (for example, the International Affective Pictures System) [27, 54]. Given this, we have demonstrated that it is possible to detect distinct patterns of hemodynamic activity generated by different affective valence elicitation tasks. Our results also provide 

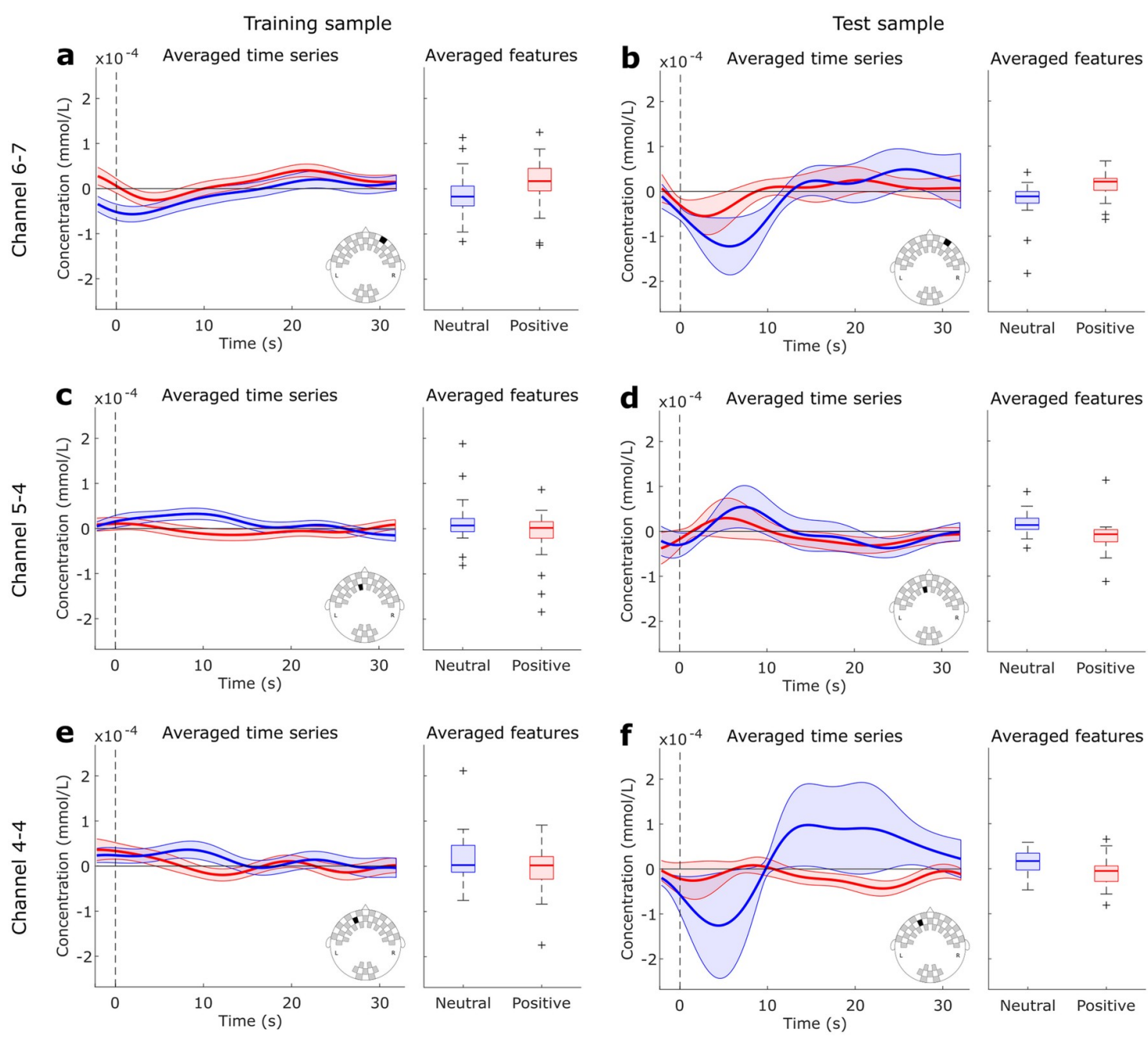

Fig 6. Averaged time series and features used as inputs to the LDA classifier during "Positive vs. Neutral" comparison in the active task. Data from Channel 6-7 is shown in (a-b), Channel 5-4 in (c-d) and Channel 4-4 in (e-f) for the training and test samples, respectively. Blue curves and boxes represent Neutral trials, and red curves and boxes Positive trials.

https://doi.org/10.1371/journal.pone.0244840.g006

evidences for the feasibility of decoding affective states in a new, untested subject, based on a model trained in previously collected and independent dataset.

For the decoding of the reactive task, only the accuracies for "Positive vs. Negative" and "Negative vs. Neutral" comparisons were higher than chance level. This finding is in line with previous inter-subject studies using the IAPS database to decode affective states, but with fMRI [13]. In a qualitative evaluation, some participants described, after the experiment, that the negative affect induced by the pictures presented during the reactive block was more intense than the positive one. Negative figures into the IAPS catalog are mainly related to death, malnutrition, sickness, poverty, and disgust [25], which are more consensual than the content of some positive stimuli, such as babies, pets or beaches. Moreover, some studies suggest that processing negative stimuli are more demanding in the brain [55], which might generate more clear signals to our classifiers than the neural processing of positive stimuli.

The decoding of the active task, on the other hand, presented significant accuracy for the "Positive vs. Neutral" condition exclusively. The participants also described that the positive imagery during active condition was more natural to achieve and more intense than the 
negative representation. This result is particularly interesting for potential neurofeedback applications $[4,56]$. For example, in psychiatric applications where patients present increased levels of negative emotions and decreased levels of positive emotions [57], improving the selfregulation between Positive and Neutral states might be clinically relevant [24].

\section{Relevant features and the neural networks of affect}

For both results in the reactive task, relevant features included both fNIRS derived oxyhemoglobin and deoxyhemoglobin concentrations measures. In fact, each chromophore is expected to provide different and complementary information. Deoxyhemoglobin is thought to be an indicator of functional activation and is more directly related to the fMRI BOLD signal [15, 58], while a recent work found a correlation between oxyhemoglobin signal and the EEG band power variation in some cortical regions [59]. Other decoding experiments also reported both hemoglobin concentrations as relevant for classification [19]. These results suggest a nonredundancy of these measures.

In accordance with two meta-analyses of neuroimaging data in affective tasks, we found that occipital areas signals were among the relevant features for classification $[28,29]$. Additionally, fNIRS studies also described activation of the occipital cortex during affective stimulation [60, 61]. Minati and colleagues, for example, found increased occipital response to positive and negative affects relative to neutral pictures of the same database we have used (IAPS) [62]. Moreover, it is interesting to note that the classifier assigned high absolute weights for a considerable number of channels from frontopolar and anterofrontal regions. A frontal lateralization of activity during the experience and regulation of emotional responses is a classical effect [63-66]. However, a broader theoretical account of affective processing, affective workspace hypothesis, states that activity patterns of the same core neural network could implement both positive and negative affective responses [26, 27]. Our application of subjectindependent affective decoding, which considers distributed brain activity, is in line with the theoretical assumptions of such model. Frontal regions, which were consistently relevant for classification in our experiments, superpose to the network nodes that have been proposed as core regions of the affective neural workspace $[28,67]$.

\section{Subject-independent designs}

fNIRS-based affective neurofeedback protocols were recently applied to healthy $[24,68]$ and psychiatric populations [69], including patients with schizophrenia [70], autism disorder [71], and attention-deficit/hyperactivity disorder (ADHD) [72, 73]. However, all these protocols have subject-specific designs which require training blocks or calibration trials for every experimental session. In addition to the expected duration of the setup preparation, these demands can act as stressors for patients presenting anxiety symptoms, or cause physical and mental fatigue shortly after the initial blocks [6]. Consequently, patients may not achieve their best performance, or the therapeutic benefits associated with the protocol. All these aspects make the investigation of subject-independent fNIRS protocols a timely research topic.

Critically, classification accuracies presented in this paper are lower than those presented in our previous subject-specific study [22]. However, the performance drop during subject-independent designs is an expected effect due to the inter-subject variability of anatomy, level of stress, rest, among others. For example, Robinson and colleagues report more than $10 \%$ of performance reduction from a subject-dependent to a subject-independent fNIRS-based motor imagery neurofeedback [74].

Also, the mean accuracy for "Positive vs. Neutral" discrimination in the active block was slightly over the $70 \%$ threshold suggested by the brain-computer interface and neurofeedback 
communities as sufficient to perform device control and communication [19,75]. This is a crucial finding since the differentiation between the active elicitation of positive affect and a neutral (resting-state) condition is the state of the art of hemodynamic-based neurofeedback protocols applied to both healthy and psychiatric populations [24, 47, 49, 50, 76, 77]. It is also important to emphasize that this result was reached using only three channels. Considering future applications, the use of only three channels means shorter setup procedure, lower instrumental and computational costs, and the possibility of even more portable systems.

\section{Limitations and future perspectives}

In subject-independent designs using fMRI data, the maximum accuracy for decoding data from new individuals is $100 \%$, and averages varying from $60 \%$ to $80 \%$ depending on the number of voxels (from 2000 to 4000) included as predictors [13]. Although our best results belong to the same accuracy range from fMRI studies, fMRI studies outperform fNIRS experiments. However, comparisons should consider the differences in the spatial resolution of both methods. While these experiments include voxels from subcortical regions, such as the amygdala [13], or even from specific neuron populations [78], affect decoding using fNIRS relies on data from external layers from prefrontal gyri $[16,17]$. This is a relevant limitation that should be considered when planning future experiments.

Despite the controls we implemented in our experimental design, collection and analysis, limitations should be recognized. First, although fNIRS signal is mainly related to the nearinfrared light absorption into the cortical surface, it also encompasses peripheral responses, including changes in skin, muscular and cranial blood flow and the aerobic process of energy consumption related to muscle contractions [16]. Consequently, some authors refer to fNIRS applications for control of devices such as corporeal machine interfaces [19]. As this constitutes one fundamental issue of this method, further validation using this approach is warranted. Here, one approach that could be explored in future studies is the use of shortseparation channels to filter systemic hemodynamic fluctuations from non-neural sources $[79,80]$.

Also, we used minimal preprocessing steps, classifying fNIRS data using the mean changes in oxy and deoxyhemoglobin concentrations as features. This approach was used in order to test fNIRS based MDB of affective state with the least assumptions possible, to avoid introducing spurious artifact in the signal and to test the feasibility for posterior real-time analysis [81, 82]. In comparison, recent studies identified that combining the mean hemoglobin concentration with other temporal and time-frequency features improves the decoding accuracies reaching values close to $90 \%$ in within-subject decoding $[19,83,84]$. Therefore, future studies should also evaluate the effect of different feature extraction techniques to the inter-participants MBD of affective states. Regarding experimental design, it is important to emphasize that potential differences in perceptual and semantic complexity of positive and negative images and of the phenomenological features of the affective states (e.g., vividness, content of memory retrieval) were not controlled in depth detail. Exploring the effects of such differences on valence decoding and investigating the possibility of disentangling such differences using subject-independent fNIRS-based classification approaches would constitute prolific lines of enquiry in affective neurofeedback.

However, even with these limitations, we reinforce the advantages of fNIRS compared to other neuroimaging techniques in implementing MBD systems. Particularly, the portability, benefit-cost relation and the good balance between spatial and temporal resolutions makes fNIRS especially suitable for this purpose [16]. In fact, these aspects create a solid base for an increasing number of studies using fNIRS for MBD of motor, cognitive, and more recently for 
decoding of affective states $[19,20,22]$. In subject-independent designs, experiments can also be found classifying semantic experiences [85] or audiovisual processing [86]. Additionally, the portability of fNIRS systems allow a fast evolution of naturalistic experiments targeting real-world applications $[87,88]$, such as affective neurofeedback systems for therapeutic purposes $[24,69]$.

\section{Conclusion}

Although more experiments are necessary to increase the classification accuracies reached here, the results from this pilot study suggest that fNIRS is a potential tool for subject-independent decoding of affective states. The accuracy in discriminating positive and neutral emotions during the affective task was significant and above the threshold desired for effective control of BCI or neurofeedback protocols. Thus, the construction of fNIRS-based subject-independent neurofeedback devices should be attempted.

\section{Supporting information}

S1 File. Supporting analysis.

(DOCX)

\section{Acknowledgments}

The authors thank Guilherme A. Z. Moraes (NIRx) and Jackson Cionek (Brain Support Brazil) for technological support.

\section{Author Contributions}

Conceptualization: Lucas R. Trambaiolli, André M. Cravo, Claudinei E. Biazoli, Jr., João R. Sato.

Data curation: Lucas R. Trambaiolli, Juliana Tossato.

Formal analysis: Lucas R. Trambaiolli, Juliana Tossato.

Funding acquisition: João R. Sato.

Methodology: Lucas R. Trambaiolli, André M. Cravo, Claudinei E. Biazoli, Jr., João R. Sato.

Writing - original draft: Lucas R. Trambaiolli, Juliana Tossato, Claudinei E. Biazoli, Jr.

Writing - review \& editing: Lucas R. Trambaiolli, André M. Cravo, Claudinei E. Biazoli, Jr., João R. Sato.

\section{References}

1. Haynes J-D, Rees G. Neuroimaging: decoding mental states from brain activity in humans. Nature Reviews Neuroscience. 2006; 7(7):523. https://doi.org/10.1038/nrn1931 PMID: 16791142

2. Kubilius J, Baeck A, Wagemans J, de Beeck HPO. Brain-decoding fMRI reveals how wholes relate to the sum of parts. Cortex. 2015; 72:5-14. https://doi.org/10.1016/j.cortex.2015.01.020 PMID: 25771992

3. Mühl C, Allison B, Nijholt A, Chanel G. A survey of affective brain computer interfaces: principles, stateof-the-art, and challenges. Brain-Computer Interfaces. 2014; 1(2):66-84.

4. Kim S, Birbaumer N. Real-time functional MRI neurofeedback: a tool for psychiatry. Current opinion in psychiatry. 2014; 27(5):332-6. https://doi.org/10.1097/YCO.0000000000000087 PMID: 25023886

5. Arns M, Batail J-M, Bioulac S, Congedo M, Daudet C, Drapier D, et al. Neurofeedback: One of today's techniques in psychiatry? L’Encéphale. 2017; 43(2):135-45. https://doi.org/10.1016/j.encep.2016.11. 003 PMID: 28041692 
6. Sulzer J, Haller S, Scharnowski F, Weiskopf N, Birbaumer N, Blefari ML, et al. Real-time fMRI neurofeedback: progress and challenges. Neuroimage. 2013; 76:386-99. https://doi.org/10.1016/j. neuroimage.2013.03.033 PMID: 23541800

7. Mariooryad S, Busso C. Exploring cross-modality affective reactions for audiovisual emotion recognition. IEEE Transactions on affective computing. 2013; 4(2):183-96.

8. Tan J-W, Andrade AO, Li H, Walter S, Hrabal D, Rukavina S, et al. Recognition of intensive valence and arousal affective states via facial electromyographic activity in young and senior adults. PloS one. 2016; 11(1):e0146691. https://doi.org/10.1371/journal.pone.0146691 PMID: 26761427

9. Lin Y-P, Wang C-H, Jung T-P, Wu T-L, Jeng S-K, Duann J-R, et al. EEG-based emotion recognition in music listening. IEEE Transactions on Biomedical Engineering. 2010; 57(7):1798-806. https://doi.org/ 10.1109/TBME.2010.2048568 PMID: 20442037

10. Bozhkov L, Georgieva P, editors. Classification models of emotional biosignals evoked while viewing affective pictures. 2014 4th International Conference On Simulation And Modeling Methodologies, Technologies And Applications (SIMULTECH); 2014: IEEE.

11. Georgieva O, Milanov S, Georgieva P, Santos IM, Pereira AT, Silva C. Learning to decode human emotions from event-related potentials. Neural Computing and Applications. 2015; 26(3):573-80.

12. Ramaraju S, Izzidien A, Roula MA, editors. The detection and classification of the mental state elicited by humor from EEG patterns. 2015 37th Annual International Conference of the IEEE Engineering in Medicine and Biology Society (EMBC); 2015: IEEE.

13. Baucom LB, Wedell DH, Wang J, Blitzer DN, Shinkareva SV. Decoding the neural representation of affective states. Neuroimage. 2012; 59(1):718-27. https://doi.org/10.1016/j.neuroimage.2011.07.037 PMID: 21801839

14. Villringer A, Planck J, Hock C, Schleinkofer L, Dirnagl U. Near infrared spectroscopy (NIRS): a new tool to study hemodynamic changes during activation of brain function in human adults. Neuroscience letters. 1993; 154(1-2):101-4. https://doi.org/10.1016/0304-3940(93)90181-j PMID: 8361619

15. Steinbrink J, Villringer A, Kempf F, Haux D, Boden S, Obrig H. Illuminating the BOLD signal: combined fMRI-fNIRS studies. Magnetic resonance imaging. 2006; 24(4):495-505. https://doi.org/10.1016/j.mri. 2005.12.034 PMID: 16677956

16. Nishitani S, Shinohara K. NIRS as a tool for assaying emotional function in the prefrontal cortex. Frontiers in human neuroscience. 2013; 7:770. https://doi.org/10.3389/fnhum.2013.00770 PMID: 24302904

17. Bendall RC, Eachus $P$, Thompson $C$. A brief review of research using near-infrared spectroscopy to measure activation of the prefrontal cortex during emotional processing: the importance of experimental design. Frontiers in human neuroscience. 2016; 10:529. https://doi.org/10.3389/fnhum.2016.00529 PMID: 27812329

18. Pinti P, Aichelburg C, Gilbert S, Hamilton A, Hirsch J, Burgess $P$, et al. A review on the use of wearable functional near-infrared spectroscopy in naturalistic environments. Japanese Psychological Research. 2018; 60(4):347-73. https://doi.org/10.1111/jpr.12206 PMID: 30643322

19. Tai K, Chau T. Single-trial classification of NIRS signals during emotional induction tasks: towards a corporeal machine interface. Journal of neuroengineering and rehabilitation. 2009; 6(1):39. https://doi.org/ 10.1186/1743-0003-6-39 PMID: 19900285

20. Hosseini SH, Mano Y, Rostami M, Takahashi M, Sugiura M, Kawashima R. Decoding what one likes or dislikes from single-trial fNIRS measurements. Neuroreport. 2011; 22(6):269-73. https://doi.org/10. 1097/WNR.0b013e3283451f8f PMID: 21372746

21. Köchel A, Plichta MM, Schäfer A, Leutgeb V, Scharmüller W, Fallgatter AJ, et al. Affective perception and imagery: a NIRS study. International Journal of psychophysiology. 2011; 80(3):192-7. https://doi. org/10.1016/j.ijpsycho.2011.03.006 PMID: 21419180

22. Trambaiolli LR, Biazoli CE, Cravo AM, Sato JR. Predicting affective valence using cortical hemodynamic signals. Scientific reports. 2018; 8(1):5406. https://doi.org/10.1038/s41598-018-23747-y PMID: 29599437

23. Hu X, Zhuang C, Wang F, Liu Y-J, Im C-H, Zhang D. fNIRS Evidence for Recognizably Different Positive Emotions. Frontiers in human neuroscience. 2019; 13. https://doi.org/10.3389/fnhum.2019.00120 PMID: 31024278

24. Trambaiolli LR, Biazoli CE, Cravo AM, Falk TH, Sato JR. Functional near-infrared spectroscopy-based affective neurofeedback: feedback effect, illiteracy phenomena, and whole-connectivity profiles. Neurophotonics. 2018; 5(3):035009. https://doi.org/10.1117/1.NPh.5.3.035009 PMID: 30689679

25. Lang PJ, Bradley MM, Cuthbert BN. International affective picture system (IAPS): Technical manual and affective ratings. NIMH Center for the Study of Emotion and Attention. 1997; 1:39-58.

26. Edelman GM. The remembered present: a biological theory of consciousness: Basic Books; 1989. 
27. Barrett LF, Bliss-Moreau E. Affect as a psychological primitive. Advances in experimental social psychology. 2009; 41:167-218. https://doi.org/10.1016/S0065-2601(08)00404-8 PMID: 20552040

28. Lindquist KA, Wager TD, Kober H, Bliss-Moreau E, Barrett LF. The brain basis of emotion: a meta-anaIytic review. The Behavioral and brain sciences. 2012; 35(3):121. https://doi.org/10.1017/ S0140525X11000446 PMID: 22617651

29. Lindquist KA, Satpute AB, Wager TD, Weber J, Barrett LF. The brain basis of positive and negative affect: evidence from a meta-analysis of the human neuroimaging literature. Cerebral Cortex. 2015; 26 (5):1910-22. https://doi.org/10.1093/cercor/bhv001 PMID: 25631056

30. Stern R, Arruda J, Hooper C, Wolfner G, Morey C. Visual analogue mood scales to measure internal mood state in neurologically impaired patients: Description and initial validity evidence. Aphasiology 1997; 11(1):59-71.

31. Tie Y, Suarez RO, Whalen S, Radmanesh A, Norton IH, Golby AJ. Comparison of blocked and eventrelated fMRI designs for pre-surgical language mapping. Neuroimage. 2009; 47:T107-T15. https://doi. org/10.1016/j.neuroimage.2008.11.020 PMID: 19101639

32. Schroeter ML, Kupka T, Mildner T, Uludağ K, von Cramon DY. Investigating the post-stimulus undershoot of the BOLD signal—a simultaneous fMRI and fNIRS study. Neuroimage. 2006; 30(2):349-58. https://doi.org/10.1016/j.neuroimage.2005.09.048 PMID: 16257236

33. Yücel MA, Selb J, Aasted CM, Lin P-Y, Borsook D, Becerra L, et al. Mayer waves reduce the accuracy of estimated hemodynamic response functions in functional near-infrared spectroscopy. Biomedical Optics Express. 2016; 7(8):3078-88. https://doi.org/10.1364/BOE.7.003078 PMID: 27570699

34. Pfurtscheller G, Schwerdtfeger A, Brunner C, Aigner C, Fink D, Brito J, et al. Distinction between neural and vascular BOLD oscillations and intertwined heart rate oscillations at $0.1 \mathrm{~Hz}$ in the resting state and during movement. PloS one. 2017; 12(1):e0168097. https://doi.org/10.1371/journal.pone.0168097 PMID: 28052074

35. Naseer N, Hong K-S. Classification of functional near-infrared spectroscopy signals corresponding to the right-and left-wrist motor imagery for development of a brain-computer interface. Neuroscience letters. 2013; 553:84-9. https://doi.org/10.1016/j.neulet.2013.08.021 PMID: 23973334

36. Naseer N, Hong MJ, Hong K-S. Online binary decision decoding using functional near-infrared spectroscopy for the development of brain-computer interface. Experimental brain research. 2014; 232 (2):555-64. https://doi.org/10.1007/s00221-013-3764-1 PMID: 24258529

37. Essenpreis M, Elwell C, Cope M, Van der Zee P, Arridge S, Delpy D. Spectral dependence of temporal point spread functions in human tissues. Applied optics. 1993; 32(4):418-25. https://doi.org/10.1364/ AO.32.000418 PMID: 20802707

38. Kothe CA, Makeig S. BCILAB: a platform for brain-computer interface development. Journal of neural engineering. 2013; 10(5):056014. https://doi.org/10.1088/1741-2560/10/5/056014 PMID: 23985960

39. Fisher RA. The use of multiple measurements in taxonomic problems. Annals of eugenics. 1936; 7 (2):179-88.

40. Thomaz CE, Gillies DF, Feitosa RQ. A new covariance estimate for Bayesian classifiers in biometric recognition. IEEE Transactions on circuits and systems for video technology. 2004; 14(2):214-23.

41. Thomaz CE, Boardman JP, Hill DL, Hajnal JV, Edwards DD, Rutherford MA, et al., editors. Using a maximum uncertainty LDA-based approach to classify and analyse MR brain images. International Conference on Medical Image Computing and Computer-Assisted Intervention; 2004: Springer.

42. Thomaz CE, Kitani EC, Gillies DF. A maximum uncertainty LDA-based approach for limited sample size problems - with application to face recognition. Journal of the Brazilian Computer Society. 2006; 12 (2):7-18.

43. Thomaz CE, Duran FL, Busatto GF, Gillies DF, Rueckert D. Multivariate statistical differences of MRI samples of the human brain. Journal of mathematical imaging and vision. 2007; 29(2-3):95-106.

44. Thomaz CE, Boardman JP, Counsell S, Hill DL, Hajnal JV, Edwards AD, et al. A multivariate statistical analysis of the developing human brain in preterm infants. Image and Vision Computing. 2007; 25 (6):981-94.

45. Sato JR, Fujita A, Thomaz CE, Martin MdGM, Mourão-Miranda J, Brammer MJ, et al. Evaluating SVM and MLDA in the extraction of discriminant regions for mental state prediction. Neurolmage. 2009; 46 (1):105-14. https://doi.org/10.1016/j.neuroimage.2009.01.032 PMID: 19457392

46. Hamilton JP, Glover GH, Hsu JJ, Johnson RF, Gotlib IH. Modulation of subgenual anterior cingulate cortex activity with real-time neurofeedback. Human brain mapping. 2011; 32(1):22-31. https://doi.org/ 10.1002/hbm.20997 PMID: 21157877

47. Johnston S, Linden D, Healy D, Goebel R, Habes I, Boehm S. Upregulation of emotion areas through neurofeedback with a focus on positive mood. Cognitive, Affective, \& Behavioral Neuroscience. 2011; 11(1):44-51. https://doi.org/10.3758/s13415-010-0010-1 PMID: 21264651 
48. Brühl AB, Scherpiet S, Sulzer J, Stämpfli P, Seifritz E, Herwig U. Real-time neurofeedback using functional MRI could improve down-regulation of amygdala activity during emotional stimulation: a proof-ofconcept study. Brain topography. 2014; 27(1):138-48. https://doi.org/10.1007/s10548-013-0331-9 PMID: 24241476

49. Zotev V, Phillips R, Young KD, Drevets WC, Bodurka J. Prefrontal control of the amygdala during realtime fMRI neurofeedback training of emotion regulation. PloS one. 2013; 8(11):e79184. https://doi.org/ 10.1371/journal.pone.0079184 PMID: 24223175

50. Young KD, Zotev V, Phillips R, Misaki M, Yuan H, Drevets WC, et al. Real-time FMRI neurofeedback training of amygdala activity in patients with major depressive disorder. PloS one. 2014; 9(2):e88785. https://doi.org/10.1371/journal.pone.0088785 PMID: 24523939

51. Hamilton JP, Glover GH, Bagarinao E, Chang C, Mackey S, Sacchet MD, et al. Effects of salience-network-node neurofeedback training on affective biases in major depressive disorder. Psychiatry Research: Neuroimaging. 2016; 249:91-6. https://doi.org/10.1016/j.pscychresns.2016.01.016 PMID: 26862057

52. Oostenveld $R$, Praamstra $P$. The five percent electrode system for high-resolution EEG and ERP measurements. Clinical neurophysiology. 2001; 112(4):713-9. https://doi.org/10.1016/s1388-2457(00) 00527-7 PMID: 11275545

53. Kuppens $P$, Tuerlinckx F, Russell JA, Barrett LF. The relation between valence and arousal in subjective experience. Psychological Bulletin. 2013; 139(4):917. https://doi.org/10.1037/a0030811 PMID: 23231533

54. Lang PJ. International affective picture system (IAPS): Affective ratings of pictures and instruction manual. Technical report. 2005.

55. Ito TA, Larsen JT, Smith NK, Cacioppo JT. Negative information weighs more heavily on the brain: The negativity bias in evaluative categorizations. Journal of personality and social psychology. 1998; 75 (4):887. https://doi.org/10.1037//0022-3514.75.4.887 PMID: 9825526

56. Birbaumer N, Ruiz S, Sitaram R. Learned regulation of brain metabolism. Trends in cognitive sciences. 2013; 17(6):295-302. https://doi.org/10.1016/j.tics.2013.04.009 PMID: 23664452

57. Gross JJ, Muñoz RF. Emotion regulation and mental health. Clinical psychology: Science and practice. 1995; 2(2):151-64.

58. Song AW, Huettel SA, McCarthy G. Functional neuroimaging: Basic principles of functional MRI. Handbook of functional neuroimaging of cognition. 2006; 2:22-52.

59. Pfurtscheller G, Daly I, Bauernfeind G, Müller-Putz GR. Coupling between intrinsic prefrontal $\mathrm{HbO} 2$ and central EEG beta power oscillations in the resting brain. PLoS One. 2012; 7(8):e43640. https://doi.org/ 10.1371/journal.pone.0043640 PMID: 22937070

60. Alpers G, Herrmann M, Pauli P, Fallgatter A. Emotional arousal and activation of the visual cortex: A near infrared spectroscopy analysis. Journal of Psychophysiology. 2005; 19(2).

61. Herrmann MJ, Huter T, Plichta MM, Ehlis AC, Alpers GW, Mühlberger A, et al. Enhancement of activity of the primary visual cortex during processing of emotional stimuli as measured with event-related functional near-infrared spectroscopy and event-related potentials. Human Brain Mapping. 2008; 29(1):2835. https://doi.org/10.1002/hbm.20368 PMID: 17315227

62. Minati L, Jones CL, Gray MA, Medford N, Harrison NA, Critchley HD. Emotional modulation of visual cortex activity: a functional near-infrared spectroscopy study. Neuroreport. 2009; 20(15):1344. https:// doi.org/10.1097/WNR.0b013e328330c751 PMID: 19738501

63. Ahern GL, Schwartz GE. Differential lateralization for positive and negative emotion in the human brain: EEG spectral analysis. Neuropsychologia. 1985; 23(6):745-55. https://doi.org/10.1016/0028-3932(85) 90081-8 PMID: 4080136

64. Ochsner KN, Gross JJ. The cognitive control of emotion. Trends in cognitive sciences. 2005; 9(5):2429. https://doi.org/10.1016/j.tics.2005.03.010 PMID: 15866151

65. Balconi M, Mazza G. Lateralisation effect in comprehension of emotional facial expression: a comparison between EEG alpha band power and behavioural inhibition (BIS) and activation (BAS) systems. Laterality: Asymmetries of Body, Brain and Cognition. 2010; 15(3):361-84.

66. Balconi M, Bortolotti A, Gonzaga L. Emotional face recognition, EMG response, and medial prefrontal activity in empathic behaviour. Neuroscience Research. 2011; 71(3):251-9. https://doi.org/10.1016/j. neures.2011.07.1833 PMID: 21840350

67. Barrett LF, Mesquita B, Ochsner KN, Gross JJ. The experience of emotion. Annu Rev Psychol. 2007; 58:373-403. https://doi.org/10.1146/annurev.psych.58.110405.085709 PMID: 17002554

68. Sakatani K, Takemoto N, Tsujii T, Yanagisawa K, Tsunashima H. NIRS-based neurofeedback learning systems for controlling activity of the prefrontal cortex. Oxygen Transport to Tissue XXXV: Springer; 2013. p. 449-54. 
69. Ehlis AC, Barth B, Hudak J, Storchak H, Weber L, Kimmig ACS, et al. Near-Infrared Spectroscopy as a New Tool for Neurofeedback Training: Applications in Psychiatry and Methodological Considerations. Japanese Psychological Research. 2018; 60(4):225-41.

70. Storchak H, Hudak J, Haeussinger FB, Rosenbaum D, Fallgatter AJ, Ehlis A-C. Reducing auditory verbal hallucinations by means of fNIRS neurofeedback-A case study with a paranoid schizophrenic patient. Schizophrenia research. 2019; 204:401. https://doi.org/10.1016/j.schres.2018.09.018 PMID: 30269928

71. Liu N, Cliffer S, Pradhan AH, Lightbody A, Hall SS, Reiss AL. Optical-imaging-based neurofeedback to enhance therapeutic intervention in adolescents with autism: methodology and initial data. Neurophotonics. 2016; 4(1):011003. https://doi.org/10.1117/1.NPh.4.1.011003 PMID: 27570790

72. Marx A-M, Ehlis A-C, Furdea A, Holtmann M, Banaschewski T, Brandeis D, et al. Near-infrared spectroscopy (NIRS) neurofeedback as a treatment for children with attention deficit hyperactivity disorder (ADHD)—a pilot study. Frontiers in human neuroscience. 2015; 8:1038. https://doi.org/10.3389/fnhum. 2014.01038 PMID: 25610390

73. Hudak J, Blume F, Dresler T, Haeussinger FB, Renner TJ, Fallgatter AJ, et al. Near-infrared spectroscopy-based frontal lobe neurofeedback integrated in virtual reality modulates brain and behavior in highly impulsive adults. Frontiers in human neuroscience. 2017; 11:425. https://doi.org/10.3389/fnhum. 2017.00425 PMID: 28928644

74. Robinson N, Zaidi AD, Rana M, Prasad VA, Guan C, Birbaumer N, et al. Real-time subject-independent pattern classification of overt and covert movements from fNIRS signals. PloS one. 2016; 11(7): e0159959. https://doi.org/10.1371/journal.pone.0159959 PMID: 27467528

75. McFarland DJ, Anderson CW, Muller K-R, Schlogl A, Krusienski DJ. BCI meeting 2005-workshop on $\mathrm{BCl}$ signal processing: feature extraction and translation. IEEE transactions on neural systems and rehabilitation engineering. 2006; 14(2):135-8. https://doi.org/10.1109/TNSRE.2006.875637 PMID: 16792278

76. Zotev V, Krueger F, Phillips R, Alvarez RP, Simmons WK, Bellgowan P, et al. Self-regulation of amygdala activation using real-time fMRI neurofeedback. PloS one. 2011; 6(9):e24522. https://doi.org/10. 1371/journal.pone.0024522 PMID: 21931738

77. Moll J, Weingartner JH, Bado P, Basilio R, Sato JR, Melo BR, et al. Voluntary enhancement of neural signatures of affiliative emotion using FMRI neurofeedback. PloS one. 2014; 9(5):e97343. https://doi. org/10.1371/journal.pone.0097343 PMID: 24847819

78. Chikazoe J, Lee DH, Kriegeskorte N, Anderson AK. Population coding of affect across stimuli, modalities and individuals. Nature neuroscience. 2014; 17(8):1114-22. https://doi.org/10.1038/nn.3749 PMID: 24952643

79. Gagnon L, Yücel MA, Boas DA, Cooper RJ. Further improvement in reducing superficial contamination in NIRS using double short separation measurements. Neuroimage. 2014; 85:127-35. https://doi.org/ 10.1016/j.neuroimage.2013.01.073 PMID: 23403181

80. Brigadoi S, Cooper RJ. How short is short? Optimum source-detector distance for short-separation channels in functional near-infrared spectroscopy. Neurophotonics. 2015; 2(2):025005. https://doi.org/ 10.1117/1.NPh.2.2.025005 PMID: 26158009

81. Coyle SM, Ward TE, Markham CM. Brain-computer interface using a simplified functional near-infrared spectroscopy system. Journal of neural engineering. 2007; 4(3):219. https://doi.org/10.1088/1741 2560/4/3/007 PMID: 17873424

82. Sitaram R, Zhang H, Guan C, Thulasidas M, Hoshi Y, Ishikawa A, et al. Temporal classification of multichannel near-infrared spectroscopy signals of motor imagery for developing a brain-computer interface. Neurolmage. 2007; 34(4):1416-27. https://doi.org/10.1016/j.neuroimage.2006.11.005 PMID: 17196832

83. Naseer N, Noori FM, Qureshi NK, Hong K-S. Determining optimal feature-combination for LDA classification of functional near-infrared spectroscopy signals in brain-computer interface application. Frontiers in human neuroscience. 2016; 10:237. https://doi.org/10.3389/fnhum.2016.00237 PMID: 27252637

84. Naseer N, Qureshi NK, Noori FM, Hong K-S. Analysis of different classification techniques for two-class functional near-infrared spectroscopy-based brain-computer interface. Computational intelligence and neuroscience. 2016; 2016. https://doi.org/10.1155/2016/5480760 PMID: 27725827

85. Zinszer BD, Bayet L, Emberson LL, Raizada RD, Aslin RN. Decoding semantic representations from functional near-infrared spectroscopy signals. Neurophotonics. 2017; 5(1):011003. https://doi.org/10. 1117/1.NPh.5.1.011003 PMID: 28840167

86. Emberson LL, Zinszer BD, Raizada RD, Aslin RN. Decoding the infant mind: Multivariate pattern analysis (MVPA) using fNIRS. PloS one. 2017; 12(4):e0172500. https://doi.org/10.1371/journal.pone. 0172500 PMID: 28426802 
87. Falk TH, Guirgis M, Power S, Chau TT. Taking NIRS-BCls outside the lab: towards achieving robustness against environment noise. IEEE Transactions on Neural Systems and Rehabilitation Engineering. 2010; 19(2):136-46. https://doi.org/10.1109/TNSRE.2010.2078516 PMID: 20876031

88. Balardin JB, Zimeo Morais GA, Furucho RA, Trambaiolli L, Vanzella P, Biazoli C Jr, et al. Imaging brain function with functional near-infrared spectroscopy in unconstrained environments. Frontiers in human neuroscience. 2017; 11:258. https://doi.org/10.3389/fnhum.2017.00258 PMID: 28567011 